IP Periodica Polytechnica

Transportation Engineering

44(2), pp. 89-96, 2016

DOI: $10.3311 /$ PPtr. 8591

Creative Commons Attribution (i)

RESEARCH ARTICLE

\section{Pavement Friction Estimation Based on the Heinrich/Klüppel Model}

\author{
Lin $\mathrm{Li}^{1}$, Kelvin C. P. Wang ${ }^{2}$, Wenting Luo ${ }^{1}$
}

Received 21 September 2015, accepted 16 November 2015

\begin{abstract}
Tire-pavement interaction is a critical analysis for conducting friction measurements and safety evaluation on highway pavements. Substantial field studies and research efforts indicate pavement friction can be predicted with tireltexture-related models (e.g. empirical or analytical models); however, developing a reliable friction prediction model for network level pavement survey still remains a challenge. In this paper Heinrich/Klüppel friction prediction model is utilized to estimate friction on Asphalt Concrete (AC) pavements. High resolution texture data are acquired from Ames high-speed profiling system, and subsequently pavement friction data are collected on the same sections with Dynatest 6875 Highway friction tester. Findings from the study indicate a good agreement between the predicted and measured Friction Numbers (FNs). It is concluded that Heinrich/Klüppel friction theory can be used as a promising surrogate for pavement safety evaluation. This study would be beneficial for complementing the existing safety evaluation methods used in highway safety program.
\end{abstract}

\section{Keywords}

Pavement Friction, Heinrich/Klüppel Model, High Friction Surface Treatment (HFST), Power Spectral Density (PSD), Fractal Analysis, Tire Penetration Depth (TPD)

\footnotetext{
${ }^{1}$ College of Transportation and Civil Engineering, Fujian Agriculture and Forestry University, Fuzhou, Fujian Province, 350002, PR China

${ }^{2}$ School of Civil and Environmental Engineering, Oklahoma State University, Stillwater OK, USA 74078
}

*Corresponding author, e-mail: kelvin.wang@okstate.edu

\section{Introduction}

One of the most important tasks of pavement engineers is to maintain safe pavements for the driving public. However, pavement safety performance would deteriorate over time due to the effects of traffic loading and environmental factors on surface texture properties (Ergun et al., 2005). As a result, potential traffic crashes may occur on pavement sections with poor frictional properties. To ensure pavements meet a certain safety requirement, a prompt and accurate measurement and evaluation of pavement frictional properties are necessary.

Generally pavement frictional properties can be assessed using either friction measurement devices or friction predictive models (ASTM E274; ASTM E303; ASTM E1911; Yandell et al., 1983; Hahn et al., 2002; Do et al., 2004; Ergun et al., 2005; Hall et al., 2009; Henry et al., 2000). Friction measurement instrument based methods are relatively complex and costly. During data collection, in most cases a truck carrying a large water tank is needed to wet pavement surface with a prescribed layer of water during measurements (ASTM E 274). Because of the limited amount of water a truck can carry, these measurements are limited to a small range, which is unsuitable for network level pavement friction measurement. Therefore, predictive models might be a promising alternative for pavement friction estimation (Yandell et al., 1983).

A large amount of work have been carried out to predict pavement friction from texture measurements using experimental methods (Leu et al., 1978; Yandell et al., 1983; Purushothaman et al., 1990; Do et al., 2004; Alvarez et al., 2005; Ergu et al., 2005; Kebrle et al., 2007, Predescu et al., 2010), but with limited successes. Studies indicated pavement texture is not the only factor influencing pavement friction, instead the predicative capability of models depends on a multitude of factors such as tire inflation pressure, temperature, rubber wear or aging, sliding velocity, vertical load, pavement texture property, and even road evenness and curviness (Klüppel et al., 2000; Persson, 2001).

Two physical friction theories are developed to investigate rubber friction and contact mechanics (Klüppel et al., 2000; Persson, 2001). The common key feature of the two theories is that they have a physical basis and predict the frictional 
coefficient of rubber materials sliding on a rough surface based on fundamental input parameters such as basic viscoelastic property of the rubber. The friction force is calculated from the dissipated energy during sliding, originating from stochastic excitations of the sliding rubber by surface asperities on various length scales. Studies indicate the predicted friction coefficients from the two theory models match well with the experimental results (Westermann, 2004).

In this paper one of the two theories, Klüppel/Heinrich model, is used to predict pavement friction since the theory model is not only related to pavement texture property, but also highly associated with tire properties. High resolution texture data is collected with Ames high speed profiling system. The predicted friction is compared with the friction data collected from Dynatest 6875 highway friction tester. Validation results indicate there is a good agreement between the predicted and measured frictions. As a result, the Klüppel/Heinrich friction model may be used as a cost-effective and promising method for the network level pavement safety surveys.

\section{Heinrich/Klüppel Friction Theory}

In Heinrich/Klüppel model, the roughness of self-affine surface or AC pavement surface can be described with three texture descriptors, namely the Hurst-Exponent (related to fractal dimension) and the other two correlation lengths parallel and perpendicular to the surface (related to the height difference correlation) (Klüppel et al., 2000). Accordingly the calculation of the three roughness descriptors involves in the fractal dimension analysis and height difference correlations.

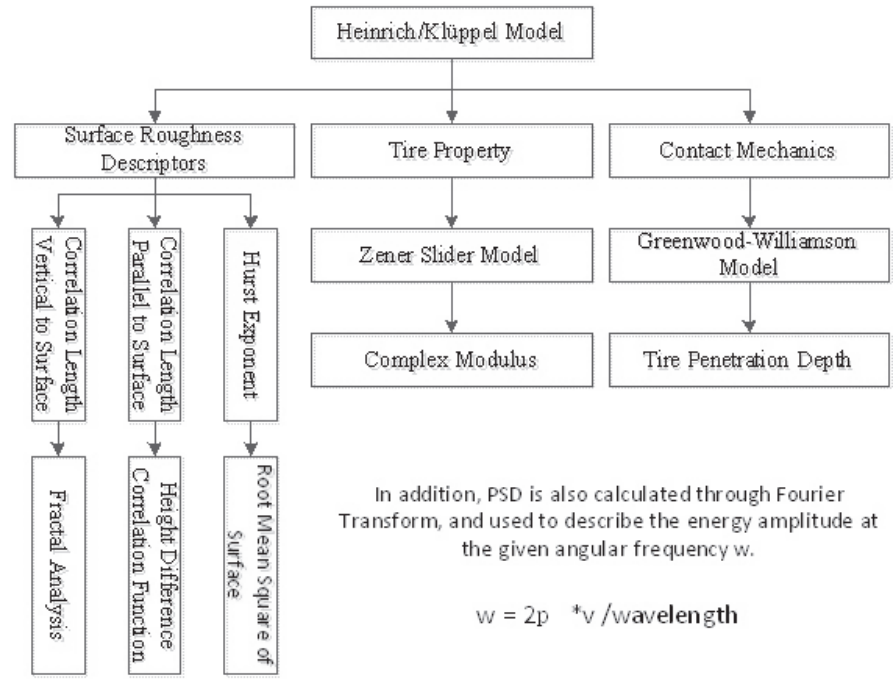

Fig. 1 Fundamental framework of Heinrich/Klüppel model

Heinrich/Klüppel friction theory describes the elastic contact between tire rubbers and pavement texture asperities using Greenwood-Williamson model (Greenwood, 1966; Klüppel et al., 2000). To predict pavement frictions, three parameters (i.e. tire penetration depth, loss modulus, and power spectral density) need to be determined based on tire properties, tire normal pressure, surface roughness, and complex viscoelastic modulus. The calculation of tire penetration depth depends on storage modulus at an excitation frequency and the correlation length parallel to pavement surface. The loss modulus is determined by relaxation time of Zener slider model, sliding velocity, and surface texture wavelengths. The power spectral density can be derived from the Fourier Transform. A deeper understanding of this theory can be referred to the original papers (Klüppel et al, 2000; Heinrich et al 2008), and the application of Heinrich/ Klüppel friction theory can be schematically described in Fig. 1.

The basic idea for the determination of the hysteresis friction coefficients is to calculate the dissipated energy $\left(E_{d}\right)$ during sliding on a rough surface. The loss energy can be mathematically described in Eq. (1) (Klüppel et al., 2000):

$$
E_{d}=\int_{0}^{V} \int_{0}^{T} \sigma(x, z, t) \dot{\epsilon}(x, z, t) d^{2} x d t
$$

Where $V$ represents the volume derived from tire deformation; $T$ represents the time to complete rubber sliding on test segment; $\sigma(x, z, t)$ represents the normal pressure over the time $t$; $\dot{\epsilon}(x, z, t)$ represents the strain over time $t$.

Per energy conservation rules, the energy dissipation per unit time must equal to the product of friction force (related to hysteretic energy loss) and the sliding velocity, then one obtains (Klüppel et al., 2000):

$$
\mu_{H K}=\frac{\left\langle z_{p}\right\rangle}{8 \pi^{2} \sigma_{0} v} \int_{w_{\min }}^{w_{\max }} w E^{\prime \prime}(w) \operatorname{PSD}(w) d w
$$

Equation (2) calculates the hysteresis friction coefficient $\mu_{H K}$ for a cylindrical rubber block undergoing a one-dimensional deformation during sliding contact at velocity $v$ with a rough surface under contact pressure $\sigma_{0} ;\left\langle z_{p}\right\rangle$ is the tire penetration depth whose calculation is related to fractal dimension and height difference correlation; $E^{\|}(w)$ is the loss energy modulus and determined by Zener slider model; $P S D(w)$ is the energy amplitude at the given angular frequency $w$ and can be determined by the Fourier Transform.

To better interpret surface texture descriptors and other parameters used in Heinrich/Klüppel friction theory model, the fractal dimension analysis, height difference correlation function, Zener slider model and tire penetration depth calculation, as well as power spectral density estimation are elaborated in the following subsections.

\section{Fractal Dimension Analysis}

The self-affine behavior is observed in the pavement texture structure or shape, leading to a natural way to describe the surface texture under various length scales (Kokkalis et al., 1998; ASME, 2009). Currently fractal concepts have already been successfully used in pavements. For instance, the use of fractals for characterizing aggregate shapes is reported 
(Carr et al., 1990); fractal number is used to represent the angularity of the particles (Li et al., 1990); the fractal concepts are also used to determine the surface area of aggregate particles (Carr et al., 1992); the fractal dimensions can be used to simulate the relationship of the surface wetting, wear and skid resistance (Kokkalis et al., 1998). In this paper fractal dimension analysis is utilized to characterize the property of pavement texture collected from Ames high speed profiling system, and the resultant fractal dimension is used to calculate one of the three roughness descriptors: Hurst exponent $(\mathrm{H})$.

Although an increasing number of papers provide a theoretical basis for observing fractal behavior in past decades (Bartlett, 1991; Mather et al., 2009), the selection of a method that can provide a consistent and reliable determination of the fractal dimension remains unsolved. Many methods, such as box counting, walking divider method, power spectrum and so on, have been developed, but most have their practical and theoretical limitations (Mather et al., 2009). In this paper the walking divider method is used to determine fractal dimension of pavement texture. This method uses a chord length (Step) to measure the number of chord lengths required to cover a fractal curve (Vallejo, 2001). The technique is based on the principle of taking rulers of varying size (Step) to cover the fractal curve and counting the number of rulers required in each case, as shown in Fig. 2.

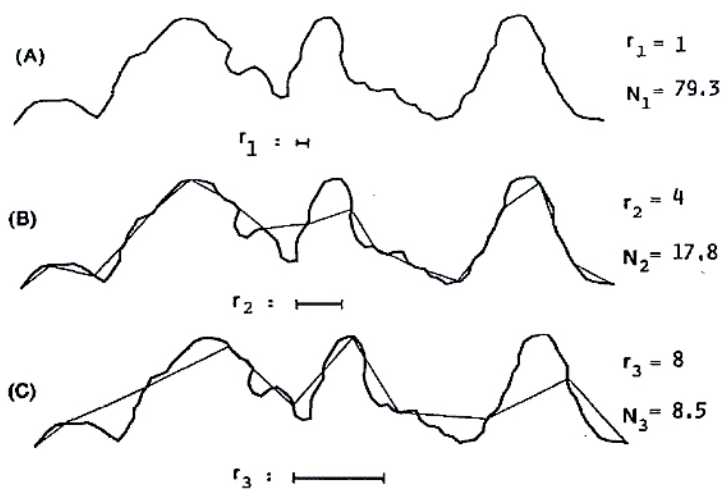

Fig. 2 The various step length (r) and number of steps $(\mathrm{N})$ required for the measured fractal curve (Vallejo, 2001)

Once the step lengths (r) and number of steps $(\mathrm{N})$ required to cover the surface texture are obtained, the fractal dimension can be calculated. Fractal Dimension (FD) equals to the ratio of the $\log$ of the number of linear elements $\mathrm{N}$ with respect to the log of the reciprocal of the linear scaling ratio (r). Its mathematical description is given in Eq. (3) (ASME, 2009).

$$
\mathbf{F D}=\frac{\log \mathbf{N}}{\log (1 / \mathbf{r})}
$$

\section{Height Difference Correlation}

Typically the roughness of a surface texture can be represented with the profile $z(x)$, the arbitrary transformation would keep the surface statistically invariant if the surface appears self-affine features, as mathematically described in Eq. (4).

$$
x \stackrel{\text { yields }}{\longrightarrow} \Lambda x, \quad z \stackrel{\text { yields }}{\longrightarrow} \Lambda^{H} z
$$

Where $\mathrm{H}$ is Hurst exponent which is related to the fractal dimension $\mathrm{D}(\mathrm{D}=2-\mathrm{H}, 0 \leq \mathrm{H} \leq 1)$.

Apart from the surface fractal dimension $\mathrm{D}$, the other two surface descriptors are also used to characterize a self-affine surface: (1) the correlation length $\xi_{\|}$parallel to the surface and (2) the correlation length $\xi_{\perp}$ perpendicular to surface. One solution to describe the two surface descriptors with the height difference correlation function, as given in Eq. (5).

$$
\operatorname{HDCF}(\lambda)=(z(x+\lambda)-z(x))^{2}
$$

Where $\langle\ldots\rangle$ denotes the average value; $\operatorname{HDCF}(\lambda)$ describes the mean square height difference of the surface at the horizontal length scale $\lambda$.

For the self-affine surface texture, the height difference $\operatorname{HDCF}(\lambda)$ would be approximating to a constant value with the increase of the length scale $\lambda$. Assume the HDCF $(\lambda)$ starts keeping invariant at the length scale $\left(\xi_{\|}\right)$, and the relationship between the length scale and correlation lengths can be mathematically described in Eq. (6) and (7).

$$
\begin{gathered}
\operatorname{HDCF}(\lambda)=\left(\frac{\lambda}{\xi_{\|}}\right)^{2} \xi_{\perp}^{2} \text { for } \lambda<\xi_{\|} \\
\operatorname{HDCF}(\lambda)=\xi_{\perp}^{2} \text { for } \lambda>\xi_{\|}
\end{gathered}
$$

From Figure 3, it can be observed that the correlation lengths $\xi_{\perp}$ and $\xi_{\|}$are determined at the length scale $\xi_{\|}$. For the length scale less than $\xi_{\|}$, the linear equation with a slope of $2 \mathrm{H}$ exists. That is, the $\xi_{\|}$can be calculated using the $\xi_{\perp}$ based on the linear equation. To calculate the $\xi_{\|}$, the $\xi_{\perp}$ needs to be determined first. Typically the correlation length $\xi_{\perp}$ is dependent on the variance of the measured height distribution of a surface, and the variance is calculated by Eq. (8).

$$
\operatorname{Var}(z)=\left\langle(z(x)-z)^{2}\right\rangle
$$

The mean height of the surface profile is represented with $\langle z\rangle$. For the self-affine pavement surface, the relationship between variance and the height correlation $\xi_{\perp}$ can be mathematically described using Eq. (9).

$$
\xi_{\perp}=\sqrt{2 \operatorname{Var}(z)}
$$




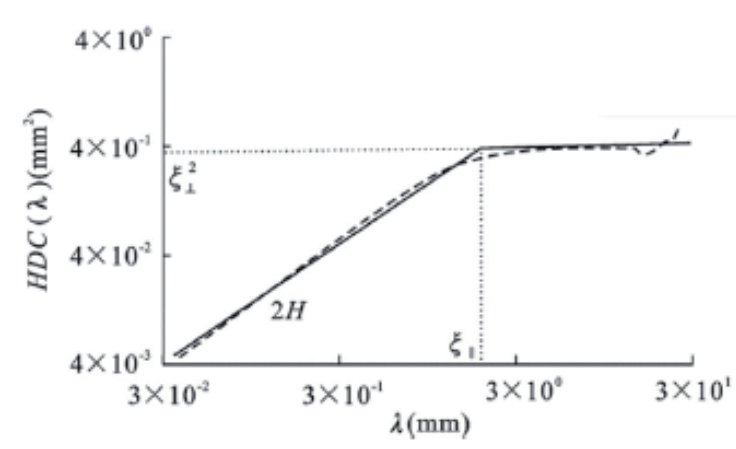

Fig. 3 Determination of the texture descriptors $\xi_{\|}, \xi_{\perp}$ and $H$

(Torbruegge et al., 2015)

Moreover, using linear equation to calculate the $\operatorname{HDCF}\left(\lambda_{\text {min }}\right)$ at the minimum wavelength scale $\lambda_{\text {min }}$, and then the correlation length $\xi_{\|}$can be calculated by Eq. (10):

$$
\frac{\xi_{\perp}^{2}-\operatorname{HDCF}\left(\lambda_{\text {min }}\right)}{\xi_{\|}-\lambda_{\text {min }}}=2 H
$$

So far the three basic elements of surface texture descriptors for self-affine surface have been determined. The accurate estimations of these three texture descriptors are critical for the prediction of pavement friction coefficients.

\section{Tire Penetration Depth (TPD)}

Aside from the three texture descriptors, the complex viscoelastic properties of the sliding rubber need to be known over a broad range of frequencies to predict the real area of contact and the energy dissipation during sliding. Heinrich/Klüppel friction theory estimates various wavelengths that contribute to the built-up of the rubber friction force (Klüppel et al., 2000). In the case of an asphalt surface, a typical range of wavelengths would be less than $200 \mathrm{~mm}$ that is associated with the contact patch length between the tire and pavement surface. The tire deformation processes lead to excitation frequencies which can reach the order of $10^{6} \mathrm{rad} / \mathrm{s}$ and higher, depending on the sliding speed and surface texture resolution.

\subsection{Zener Slide Model}

To describe the viscoelastic behavior of the rubber, a Zener slider model (also termed standard linear solid model), is introduced. The Zener slider model is a generalization of the simple constant spring and dashpot models, as illustrated in Fig. 4. Its complex modulus can be described in Eq. (11).

$$
\begin{aligned}
E(\omega) & =E^{\prime}(\omega)+i E^{\prime \prime}(\omega) \\
& =\frac{E_{e}+\left(E_{m}+E_{e}\right) \omega^{2} \tau_{z}^{2}}{1+\omega^{2} \tau_{z}{ }^{2}}+i \frac{E_{m} \omega \tau_{z}}{1+\omega^{2} \tau_{z}{ }^{2}}
\end{aligned}
$$

Where $\tau_{z}=\eta / E_{m}$ is the characterized relaxation time of the Zener slider; $E_{e}$ is the elastic modulus of the spring in parallel with the damping having viscosity modulus of $\eta$ (see Fig. 3); $E_{m}$ is elastic the modulus of the spring in series with the damping (see Fig. 3); the angular velocity $\omega=2 \pi v / \lambda$ depends on the sliding velocity and surface texture wavelength.

The rubber storage and loss modulus are denoted by $E^{\prime}(\omega)$ and $E^{\prime \prime}(\omega)$, respectively, which are related to sliding velocity, surface texture wavelength distribution, and the three modulus $\left(E_{e}, E_{m}, \eta\right)$ in Zener slider model.

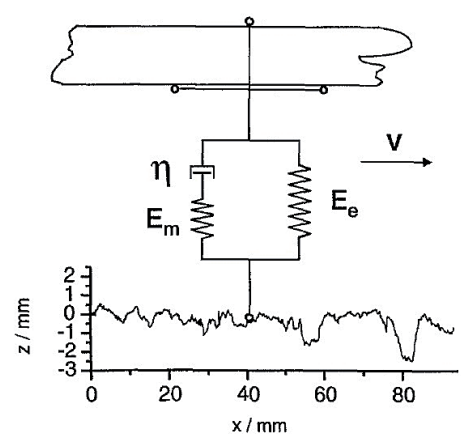

Fig. 4 Diagram of the basic elements of Zener slider model (Klüppel et al., 2000)

\subsection{TPD Estimation}

The elastic contact between the rubber tire and pavement surface typically occurs at the summits of the highest asperities, as described in Fig. 5. The $d$ is the distance between the rubber surface and pavement surface, and $\phi_{z}$ is the normalized distribution function of the surface summits.

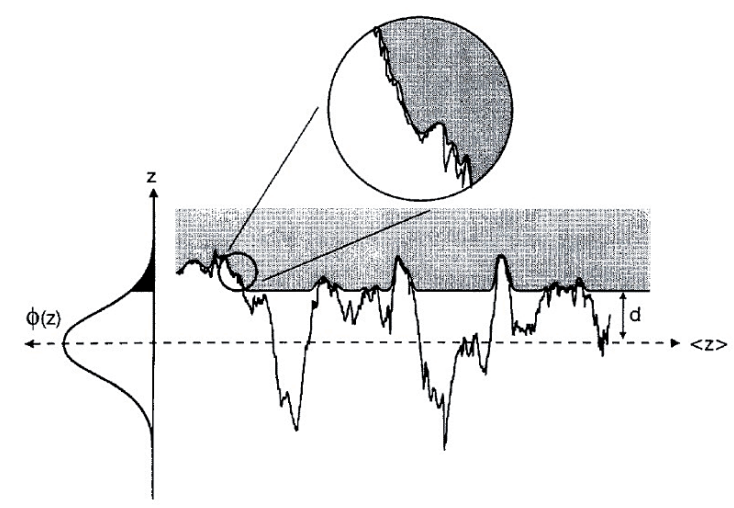

Fig. 5 Schematic representation of elastic contact between a rubber and rough surface (Klüppel et al., 2000)

Greenwoods and Williamson (GW) develop a rough surface contact theory which can be used to evaluate the mean penetration depth into the asperities, involving in the variance of the surface height $\left(m_{0}\right)$, square mean slope of surface height $\left(m_{2}\right)$, and the curvature of the height $\left(m_{4}\right)$. Their mathematical descriptions are given in Eq. (12), (13), and (14), respectively.

$$
m_{0}=\operatorname{Var}(z)
$$




$$
\begin{aligned}
& m_{2}=\left\langle\left(\frac{d z}{d x}\right)^{2}\right\rangle \\
& m_{4}=\left\langle\left(\frac{d^{2} z}{d^{2} x}\right)^{2}\right\rangle
\end{aligned}
$$

The statistical parameter of the GW theory $\alpha=m_{0} m_{4} / m_{2}{ }^{2}$ which determines the variance of the surface summit distribution and the asperity density of the surface. The surface summit distribution $\delta_{S}$ and the distance $d$ between two surfaces can be determined by $\alpha$, as described by Eq. (15) and (16):

$$
\begin{gathered}
\delta_{s}=\left(1-\frac{0.8968}{\alpha}\right) m_{0} \\
d=4 \sqrt{\frac{m_{0}}{\pi \alpha}}
\end{gathered}
$$

The GW theory can be expressed by the normalized distribution function $\phi_{z}$ of the profile $\mathrm{z}(\mathrm{x})$, and the surface height variance $\sigma$, height mean $\mu$, and the mean distance $d$, given in Eq. (17) and (18):

$$
\begin{gathered}
\phi_{Z}=\frac{1}{\sigma \sqrt{2 \pi}} e^{-\frac{(z-\mu)^{2}}{2 \sigma^{2}}} \\
F_{1}\left(\frac{d}{\sigma}\right)=\int_{d / \sigma}^{\infty}\left(z-\frac{d}{\sigma}\right) \frac{1}{\sigma \sqrt{2 \pi}} e^{-\frac{(z-\mu)^{2}}{2 \sigma^{2}}} d z
\end{gathered}
$$

Based on Eq. (11)-(18), the relationship between the mean penetration depth and the normal stress can be expressed in Eq. (19):

$$
\left\langle z_{p}\right\rangle=\tilde{\sigma} F_{1}\left(\frac{d}{\sigma}\right)
$$

\section{Power Spectral Analysis}

Power spectral analysis is used to calculate the spectrum density of various wavelengths of pavement texture, which is a well-known method for the interpretation of complicated signals containing a variety of wavelengths and amplitudes (Sayers et al., 1996). In order to investigate the power energy intensity at various wavelengths, typically two methods are available (Karamihas, 2005). One method is based on its autocorrelation function, which can be obtained by converting the integral calculation to the sum of the sampled elevation points for digitalized discrete road profiles. The other method is based on the Fourier Transform (FT) which can be obtained by computing the amplitudes of the sinusoids that are decomposed from the road texture, as given in Eq. (20). The output of the transformation represents squared amplitude at each frequency of the original data in the frequency domain. When scaled in this manner, this transform is called a PSD function (Abbas et al., 2007; Sayers et al., 1998).

$$
\mathrm{F}(k)=\sum_{m=0}^{N-1} f(m) e^{-i 2 \pi\left(\frac{k m}{N}\right)}
$$

Where $N$ represents the pavement profile length, $f(m)$ is the height value at pixel $m$ along the profile; $F(k)$ is the transformed amplitude at frequency $k$ or wave number $k$.

\section{Case Study}

\subsection{Field Test}

In this study one example is taken to demonstrate the application of the friction prediction theory in pavement safety surveys. The data collection is conducted on South Dakota (SD)-I 229, starting at GPS coordinate of 43.531788, -96.697639, and ending at GPS coordinate of 43.537987, -96.693893, with a total length of approximately 820 meters. The data collection site is the curve crossing Southeastern Ave. and E 18th Ave, as shown in Fig. 6.

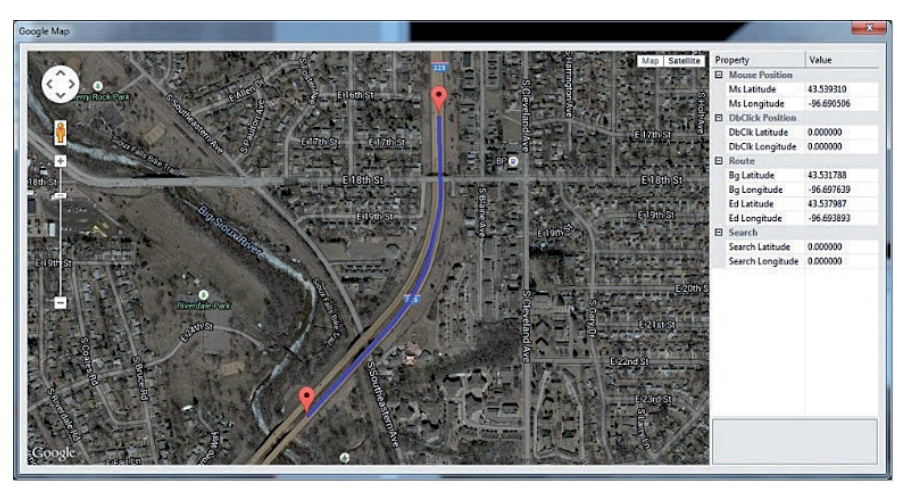

Fig. 6 Texture data plotting on the test section

The test section consists of two surface types: High Friction Surface Treatment (HFST) and the regular AC pavement surface type. For the pavement friction measurements, the HFST segment has a length of approximately $560 \mathrm{~m}$. The regular surface includes the lead-in and lead-out segments, with the lengths of $121 \mathrm{~m}$ and $118 \mathrm{~m}$ respectively. One line-of-sight texture data is also collected on the same section, with a sample interval of $0.25 \mathrm{~mm}$, as illustrated in Fig. 7. It should be noted that the texture data and the friction data have the same lead-in and lead-out segments.

\subsection{Comparison and Validations}

To better compare and validate the data with adequate number of samples, the test section would be sampled into 53 segments in this study. Each segment has a length of $15.4 \mathrm{~m}$. The HFST section starts at the location of approximately Sample\#8 and ends at the location of approximately Sampe\#46, as delineated in Fig. 8. To ensure the reliability of the friction data, three repetitive measurements are made on the test route, as illustrated in Fig. 8. It can be observed that the three measurements have the excellent agreements with each other, with the correlation coefficients of $0.97,0.96$, and 0.99 , respectively. In this study the 


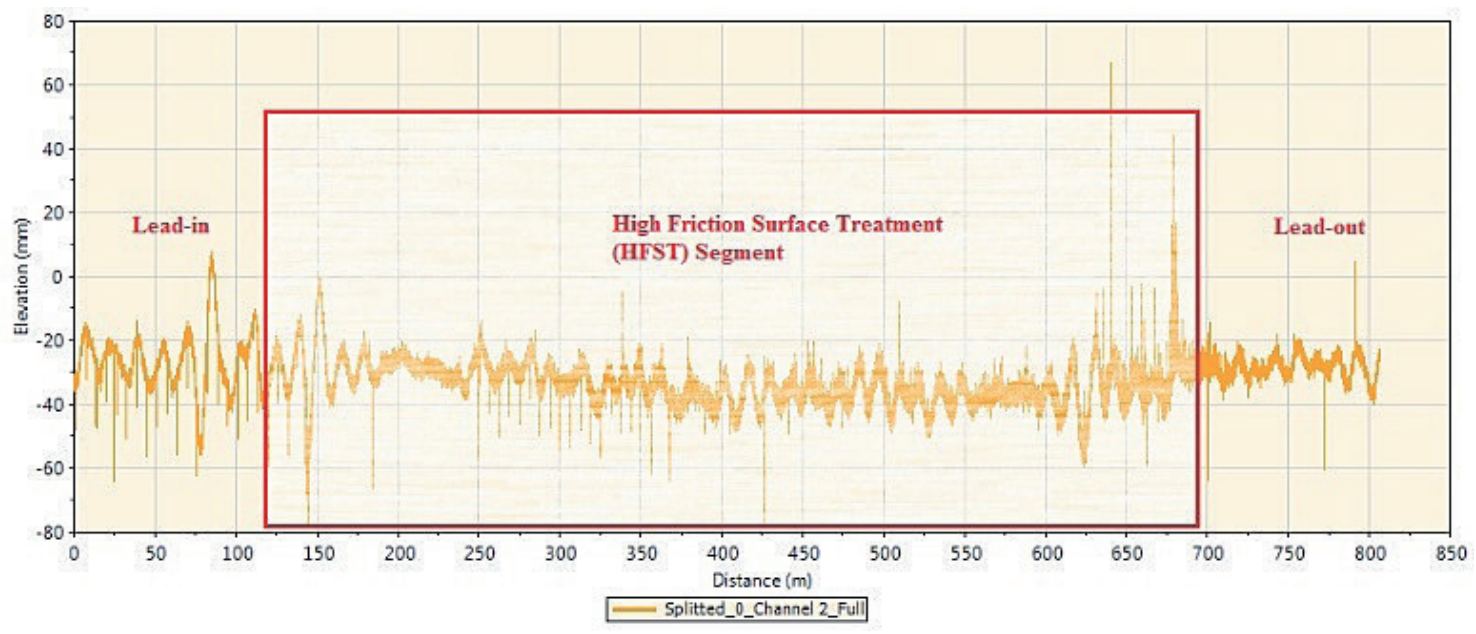

Fig. 7 SD-I 229 field test site

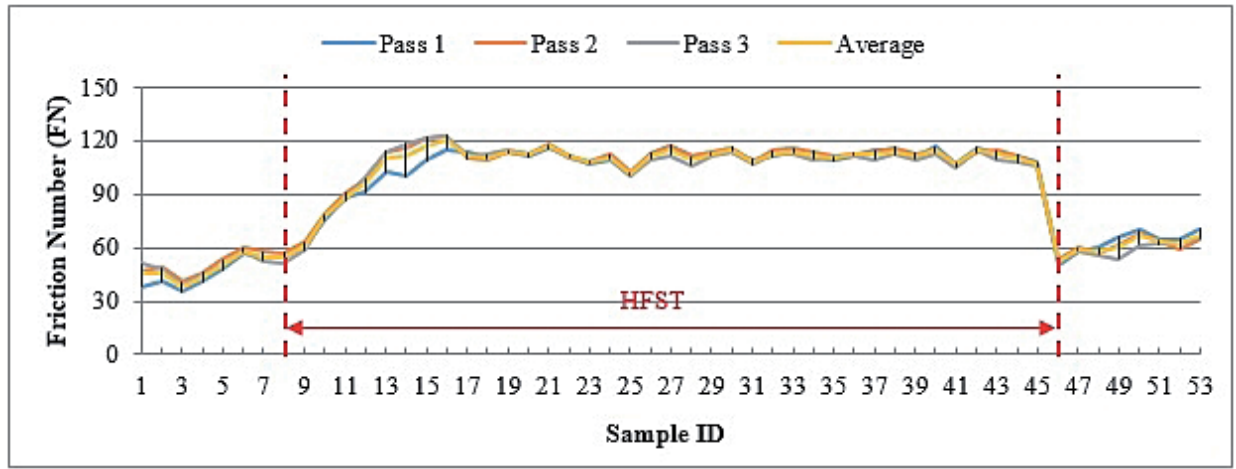

Fig. 8 Three repetitive friction measurements on SD-I 229

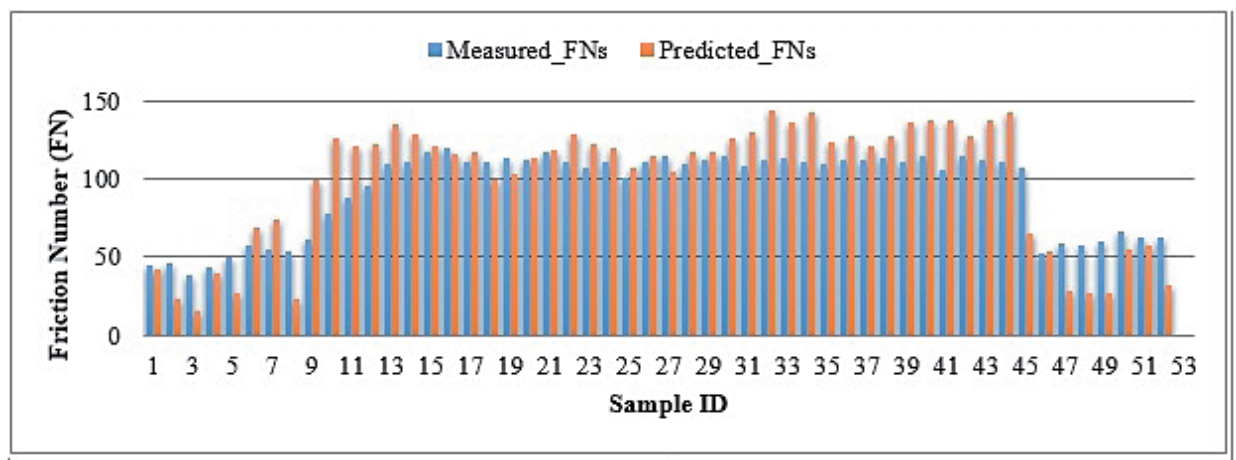

Fig. 9 Comparison between the measured and predicted FNs

mean friction values from the three passes or measurements are served as the basis for the comparison and validation with the predicted frictions from Heinrich/Klüppel model.

Heinrich/Klüppel friction theory is used to predict the pavement friction values based on the collected texture data. Through a number of data processing techniques presented in this study, the pavement friction numbers along test section are estimated, as given in Fig. 9. Note that the agreements between the predicted and measured FNs are not as good as that shown in Fig. 8; and the predicted frictions are underestimated on the lead-in and lead-out segments, while overestimated on the
HFST segment. In addition, the apparent differences appear at the transition segments between the regular pavement surface and HFST such as Samples \#9 and \#47.

To describe the relationships between the measured and predicted FNs, the linear regression analysis is used. The correlation results between the predicted and measured FNs are plotted in Fig. 10. Note that generally the FNs at the regular segments are less than 60, while the FNs at the HFST segment are greater than 100 . Findings indicate the good correlation between the measured and predicted FNs $R^{2}=0,79$ is observed, as described in Fig. 10. 


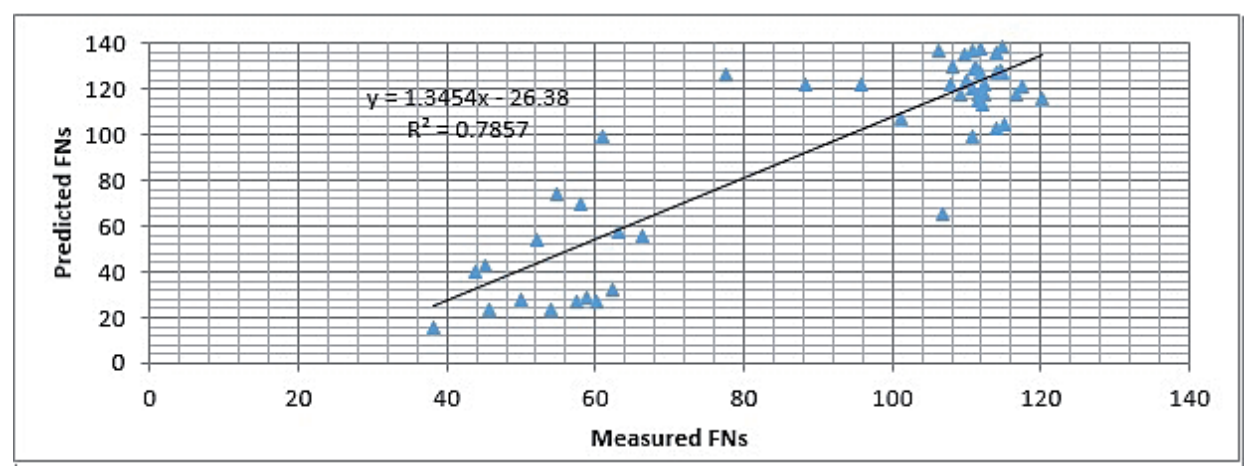

Fig. 10 Linear regression analysis between the predicted and measured FNs

\section{Conclusions and Recommendations}

This study aims to estimate the hysteresis friction coefficients of pavements by calculating the dissipated energy during sliding on a rough surface. The estimation of dissipated energy over a distance needs to deal with surface texture roughness, the rubber properties, and the contact mechanics between rubber surface and pavement surface. In the Heinrich/Klüppel model, Hurst Exponent, height difference correlation lengths parallel and perpendicular to pavement surface are used to describe surface texture roughness. Their calculations depend on fractal analysis and height difference correlation function. Subsequently the Green- Williamson model is used to describe the elastic contact between rubber and rough surfaces, which typically occurs at the summits of the highest asperities. PSD is used to calculate the spectrum density of various wavelengths of pavement texture. Finally an example is taken to illustrate the application of Heinrich/Klüppel model on pavement friction prediction. Results indicate that a good agreement is observed between the predicted and measured FNs. This study would be beneficial for complementing existing safety evaluation methods used in highway safety program.

For the purpose of the project- and network- level safety surveys, more field validation work need to be conducted on various AC pavements to improve the reliability of the model as follows:

1. The area texture data is recommended to replace the oneline-sight texture data, such as the new 3D lane-width $1 \mathrm{~mm}$ systems, for more accurate prediction of pavement frictions, especially for irregular pavement surfaces. At least, the multiple measurements should be conducted on the same test section for the one-line-sight data acquisition systems;

2. Data processing techniques need to be further investigated to remove the noises of texture data, determine the wavelengths of interest, and calculate the related parameters used in the Heinrich/Klüppel model;

3. The initialization of the variables needs to be validated, such as the modulus of the rubber block and the tire pressure.

4. The texture data resolution is highly associated with the prediction of pavement frictions, and thus higher resolution data in both vertical and horizontal directions may be necessary.

\section{Acknowledgement}

The research has been supported by FHWA EN-15-RS-101 "3D Laser Imaging based Real Time Pavement Surface Evaluation for High Friction Surfacing Treatments (HFST)". Efforts by David Merritt are appreciated in supplying the research team test data.

\section{References}

Abbas, A., Kutay, M. E., Azari, H., Rasmussen, R. (2007) Three Dimensional Surface Texture Characterization of Portland Cement Concrete Pavements. Computer Aided Civil and Infrastructure Engineering. 22(3), pp. 197-209.

Alvarez, L., Yi, J., Horowitz, R., Olmos, L. (2005) Dynamic friction modelbased tire-road friction estimation and emergency braking control. Journal of Dynamic Systems, Measurement, and Control. 127(1), pp. 22-32. DOI: $10.1115 / 1.1870036$

American Society for Testing and Materials (ASTM) (2011) ASTM E 274: Standard Test Method for Skid Resistance of Paved Surfaces Using a Full-Scale Tire. ASTM Standard Practice E 274 Book of ASTM Standards. Volume 04.03 Philadelphia.

American Society for Testing and Materials (ASTM) (2011) ASTM E 303: Standard Test Method for Measuring Surface Frictional Properties Using a British Pendulum Tester. ASTM Standard Practice E 303 Book of ASTM Standards. Volume 04.03 Philadelphia.

American Society for Testing and Materials (ASTM) (2011) ASTM E 1911: Standard Test Method for Measuring Paved Surface Frictional Properties Using Dynamic Friction Tester. ASTM Standard Practice E 1911 Book of ASTM Standards. Volume 04.03 Philadelphia.

ASME (2009) Surface Texture (Surface Roughness, Waviness, and Lay), ASME B46.1, American Society of Mechanical Engineers. New Work: Three Park Avenue.

Bartlett, M. L. (1991) Comparison of methods for measuring fractal dimension. Australian Physical and Engineering Science in Medicine. 14(3), pp. 146-52.

Carr, J. R., Norris, G. M., Newcomb, D. E. (1990) Characterization of aggregate shape using fractal dimension. TRB, 69th Annual Meeting. Washington DC.

Carr, J. R., Mirsa, M., Litchfield, J. (1992) Estimating surface area for aggregate in the size range: one millimeter or larger. TRB, 71st Annual Meeting. Washington DC.

Do, M. T., Marsac, P., Delanne, Y. (2004) Prediction of Tire/Wet Road Friction from Road Surface Microtexture and Tire Rubber Properties. In: SURF 2004-5th Symposium on Pavement Surface Characteristics. pp. $13 \mathrm{p}$-graphiques. 
Ergun, M.D., Lyinam Sukriye, Lyinam A.F. (2005) Prediction of Road Surface Friction Coefficient Using only Macro- and Micro texture Measurement. Journal of Transportation Engineering. 131(4), pp. 311-319.

DOI: $10.1061 /($ asce $) 0733-947 \times(2005) 131: 4(311)$

Greenwood, J. A., Williamson, J. B. P. (1966) Contact of nominally flat surfaces. In: Proceedings of the Royal Society of London A: Mathematical, Physical and Engineering Sciences. 295(1442), pp. 300-319.

Hahn, J. O., Rajamani, R., Alexander, L. (2002) GPS-based real-time identification of tire-road friction coefficient. IEEE Transactions on Control Systems Technology. 10(3), pp. 331-343. DOI: 10.1109/87.998016

Hall, J. W., Smith, K. L., Titus-Glover, L., Wambold, J. C., Yager, T. J., Rado, Z. (2009) NCHRP web-only document 108: Guide for pavement friction. Transportation Research Board of the National Academies. Washington, DC.

Heinrich, G., Klüppel, M. (2008) Rubber friction, tread deformation and tire traction. Wear. 265(7-8), pp. 1052-1060. DOI: $10.1016 /$ j.wear.2008.02.016

Henry, J. J. (2000) Evaluation of pavement friction characteristics. (291). Transportation Research Board.

Karamihas, S. M. (2005) Critical profiler accuracy requirements.

Kebrle, J., Walker, R. (2007) Texture measurement and friction estimation using laser data acquisition and neural networks. In: Proceedings of the 9th WSEAS.

Klüppel, M., Heinrich, G. (2000) Rubber friction on self-affine road tracks. Rubber chemistry and technology. 73(4), p. 578-606. DOI: $10.5254 / 1.3547607$

Kokkalis, A., Panagouli, O. (1998) Fractal Evaluation of Pavement Skid Resistance Variations. I: Surface Wetting. Chaos, Solitons \& Fractals. 9 (11), pp. 1875-1890. DOI: 10.1016/S0960-0779(97)00138-0

Leu, M. C., Henry, J. J. (1978) Prediction of skid resistance as a function of speed from pavement texture measurements. Transportation Research Record. 666, pp. 7-13.
Li, L., Chan,P., Zollinger, D.G., Lytton, R.L. (1990) Quantitative analysis of aggregate shape based on fractals. ACI Materials. 90(4), pp. 357-365. DOI: $10.14359 / 3894$

Mather, P., Brandt, Tso (2009) Classification methods for remotely sensed data. CRC press.

Persson, B. N. (2001) Theory of rubber friction and contact mechanics. The Journal of Chemical Physics. 115(8), p. 3840-3861. DOI: $10.1063 / 1.1388626$

Predescu, A., Pascovici, M. D., Cicone, T., Popescu, C. S., Grigoriu, C., Dragulinescu, D. (2010) Friction evaluation of lubricated laser textured surfaces. Lubrication Science. 22(10), pp. 431-442.

DOI: $10.1002 / 1$ s. 123

Purushothaman, N., Heaton, B. S., Moore, I. D. (1990) A numerical Analysis of the Friction Mechanism of Grooved Road Surfaces. Surface Characteristics of Roadways: International Research and Technologies, ASTM STP. 1031, pp. 127-137. DOI: 10.1520/stp23358s

Sayers, M. W., Karamihas, S. M. (1998) The little book of profiling. 2. The Regent of the University of Michigan.

Torbruegge, S., Wies, B. (2015) Characterization of pavement texture by means of height difference correlation and relation to wet skid resistance. Journal of Traffic and Transportation Engineering (English Edition). 2(2), pp. 59-67. DOI: 10.1016/j.jtte.2015.02.001

Vallejo, L. E. (2001) Fractal Assessment of the Surface Texture of Pavements. International Journal of Pavement Engineering. 2(2), pp. 149-156. DOI: 10.1080/10298430108901723

Westermann, S., Petry, F., Noes, R. (2004) Experimental investigations into the predictive capabilities of current physical rubber friction theories. Kautschuk Gummi Kunststoffe. 57(12), pp. 645-650.

Yandell, W. O., Taneerananon, P., Zankin, V. (1983) Prediction of Tire/Road Friction from Surface Texture and Tread Rubber Properties. Frictional Interaction of Tire and Pavement. pp. 304-322. DOI: 10.1520/stp28530s 